\title{
Developing Internet Based Teaching and Learning Resources for Social Sciences: A CSCW approach.
}

\begin{abstract}
Introduction
The Internet has awesome potentiality as a global network of information and the world wide web provides a potentially efficient and effective protocol for the delivery of information. The content of the resources made available are often of particular interest to those in university
\end{abstract} settings engaged in teaching social sciences. Technologies will most probably continue to be innovative and technical advances will increase access to these resources. Whilst this will be welcomed progress it also raises a series of issues and problems especially with regards to using Internet based resources in social science university curricula.

In this paper I will argue that whilst the use of Internet resources is potentially good for university teaching and learning, the development of these resources has often been rather haphazard. The driving forces behind the development of these resources have been either from enthusiasts or technical specialists. My argument is that the Internet is too precious and important a resource to be allowed to develop in such an unmanaged fashion. I will be advancing an argument that calls for the incorporation of non-technical knowledge in development of Internet based resources that are appropriate for use in university social science teaching and learning environments.

The ideas that I intend to express are to a large extent exploratory and intended to be evocative rather than the last word on the subject. My arguments are initially premised upon the conception that the Internet can be considered simply as a piece of information technology. The Internet is a global network of information which provides a potentially efficient and effective protocol for the delivery of information. In this regard the Internet is special but if we consider the popular image of the Internet as a 'super highway' then it is possible to think of it as a giant communications technology. Whilst the Internet has some specific features I wish to contend that if it is simply considered as a communications technology the issues that pervade its use in social science teaching also pervade the use of other information technologies in this area. Given this, more general arguments about information technology and social science teaching also apply to Internet based resources.

\section{The incorporation of information} technology into teaching settings generally proceeds from a 'technology is obviously a good thing' approach. In some quarters this is considered as axiomatic. Whilst I am sympathetic to the idea of incorporating technology into higher education teaching and learning it is the abandoning of this axiom that is essential if information technology is to be successful. The motivation behind information technology in the university setting has been from, enthusiasts on the one hand and technical specialists on the other. I will refer to this as the 'technologist perspective'.

My argument is that the 'technology is obviously a good thing' approach is a flawed departure point. Due to the hegemonic domination of technical expertise held within the technologist perspective the design and implementation of new information technologies in teaching and learning environments will have very limited success. I will be advancing an argument against the 'technologist' approach that calls for the incorporation of non-technical knowledge in technological developments in university teaching and learning. This will draw upon some of the advances that have come out of Computer Supported Cooperative Work (CSCW) approaches. I will also argue that a particular theoretical conception of 'work' and of the role of social science in technological design and implementation is appropriate.

Technology and Teaching Social Science Part of my disquiet with the technologist perspective's approach to the design and implementation of new information technology in teaching and learning environments is the generality of the argument that technology is necessarily a good thing. The lack of specificity in this approach engenders a poor understanding of the particularities of university teaching and learning. In much the same way it would be easy to talk quite generally about technology and university teaching and learning, but to avoid this pitfall I will confine this discussion to the social sciences in particular.

In the social sciences, students' experiences of information technology will mostly be in the form of desk top computing of the PC or Macintosh variety. Their introduction to computing will often form part of research 
methods or study skills. The role of the computer in this instance is often not to deliver computer based or assisted learning but rather software and hardware are used as tools to undertake tasks rather than as learning technologies.

In the British context, despite funding being directed towards computer based or assisted learning, in the social sciences there has been an absence of completed and useable bespoke software packages. The failure of these endeavours is evident insofar as there are few examples that are routinely incorporated into social science curricula. These bespoke software packages are not in widespread use in departments in British higher education!

The alternative to bespoke software is the re-use of existing technology. In these endeavours software, and to a much lesser extent hardware, are directed toward a teaching and learning requirement in an attempt to add value to a specific learning experience. From my own experience and that of colleagues, these attempts to re-use existing technology are at best problematic. The re-used existing technology, is generally attempted on an ad hoc basis and its development is time consuming, and labour intensive. In the majority of cases the development has not proceeded from a clear pedagogical requirement and the end products lack the sophistication required to deliver the high quality educational experience that is the hall mark of universities. The 'value added' nature of these endeavours is not necessarily tractable, especially when cost is entered into the equation.

Despite the huge potentiality of technology for social science teaching and learning, attempts to introduce bespoke software and to re-use existing technology are ill conceived. This is due to the taken for granted assumptions about the actual nature of the teaching and learning environment and the lack of a comprehensive empirical understanding of the processes that are in motion. This in turn leads to a shallow understanding of the consequentiality of the introduction of new computer based technologies in social science teaching.

It is highly likely that a similar unsatisfactory situation could arise with Internet based resources. The Internet offers social science teachers and students a global network of information resources. Internet based resources are potentially good for university teaching and learning but the development of these resources has often been rather haphazard. Once again the driving forces behind the development of these resources have been either from enthusiasts or technical specialists. The Internet is too precious and important a resource to be allowed to develop in such an unmanaged fashion.

In the world of commerce and industry many technical endeavours which are based around personal computing have not furnished adequate results. This has been due the technology failing to pay sensitive account to what I shall term as the 'innate sociality' of the environments into which they are being introduced. This parallels the situation in social science teaching in universities. One solution to the problem of new technology and the workplace has been the development of Computer Supported Cooperative Work (CSCW) as a design paradigm. I do not wish to argue that the work environment in the world of commerce and industry is the same as it is in higher education, although there are some obvious similarities at a generic level. I maintain that a particular theoretical sociological conception of 'work' can inform CSCW design and this is appropriate to the design and implementation of computer based learning technologies in general and this extends to the development of Internet based resources for social science teaching.

In the next part of the paper I will introduce the idea of Computer Supported Cooperative Work (CSCW). The material relates to the development of technology more generally and is not restricted to either teaching and learning or the internet. This will provide a context within which my position can later be developed.

\section{The Problem of Human Computer Interaction}

Fundamental to an understanding of the propriety of CSCW is an appreciation of the problems of a Human Computer Interaction (HCI) approach. HCI was a new and radical approach to systems design that achieved prominence in the 1980's and it sought to provide a better cognitive coupling between human users and computers (Bannon 1989). CSCW can reasonably be considered as a response to the failings of Human Computer Interaction (HCI) approaches.

HCI is a general framework for innovation aimed at developing interaction techniques, analysis methods, software and computer systems within a controlled context in order to create enhanced products. HCI endeavoured to go beyond simply providing improved surface characteristics, and hoped to address wider issues surrounding human interaction with computers. In this sense $\mathrm{HCI}$ is a design and engineering science as it aims to produce artefacts of hardware and software within satisfactory frameworks of compromise that take functionality, performance and cost into account (Brooks 1990).

The HCI perspective recognised that there were human consequences to the introduction of new technology, and that how technology was developed and applied could profoundly affect work. With regard to the introduction of new technology questions of usability, applicability and acceptability were being raised. From within the HCI camp these issues were viewed as being of legitimate concern. What was considered necessary was an applied psychological dimension located within a problem centred approach which would enable HCI practitioners to 
undertake research that would inform future designs ${ }^{1}$ (Blacker \& Osborne 1987).

Despite HCI being a radical new initiative the organisation of work is in fact endlessly richer and more complex than the majority of formal psychological models could have conveyed. Due to the rigid frameworks that such systems imposed, human actors were not furnished with sufficient flexibility to make the system function (Bannon 1989). Another draw back which stems from the psychological foundation is that $\mathrm{HCI}$ fashioned itself as a general paradigm for innovation and design in limited and controlled environments (Brooks 1990). Much of the early HCI work was confined to rather small scale controlled experiments with the presumption that the findings could be generalised to other settings (Barnard and Grudin 1988). The hoped for contribution of HCI to the design of computer systems and novel interfaces did not materialised in the 1980's (Carroll 1987). Gray and Atwood (1988) note the lack of examples of developed HCI systems. This is largely due to the inherent deficiencies in HCI approaches and what is required is an alternative theoretical and methodological orientation (Luff \& Heath 1990).

\section{Computer Supported Cooperative Work}

The expression CSCW is a comparatively new one in the information technology vocabulary and was first coined in the mid 1980's by researchers in the USA. The term was most notably used by Greif (1988) and has been applied as an umbrella term which takes in anything to do with computer support for activities involving more than one person. An alternative terminology to $\mathrm{CSCW}$ includes the expressions 'groupware' and 'workgroup computing' (Clark and O’Donnell 1991).

CSCW is very much a generic term. Bannon (1990) argues that despite disagreements about specific detail most CSCW practitioners would agree with Lyytinen (1989) who asserts that CSCW is an attempt to place emphasis on both the distinctive qualities of cooperative work processes and on questions of systems design.

CSCW takes from its point of departure the visibly processual character of social activity (Harper \& Randall 1992). The organisation of situated action is an emergent property of the interactions between actors and their environments (Suchman 1987). Settings engender a specificity unique to their social organisation. Sociological inquiry within CSCW must not be ad hoc or abstract and divorced from examination of the specificity of the setting. We must attempt to study settings and explore the coordinated tasks that computers might support in the context and settings which they occur (Luff and Heath 1990). It is fundamental to examine the natural settings where tasks and activities exhibit their sociality (Bannon \& Schmidt 1991).
The role of an effective CSCW system as its name suggests is to support the cooperative nature of work. Hirscheim and Klien (1989) assert that the good system must not be designed in what they term as the 'usual sense', but has to be designed and developed within the framework of the social interactions that are embedded in the environment in which the technology is to be incorporated. The caveat that must be issued here is that in no sense is there an objective set of criteria that form a typology for an effective system. The system must be developed within what they term as the 'user's perspective'. A CSCW system is not however simply an electronic cloning or duplication of a working environment. In contrast it is a pragmatic attempt to support the cooperative tasks of work in context within its natural social and physical environment.

In terms of sociological inquiry, ethnography is the tool of sociological research most applicable in CSCW

endeavours. As with all research methods ethnography has advantages and disadvantages but the potency, in the CSCW context, is that it depicts the activities of social actors from their own perspective. This challenges the preconceptions that alternative social science approaches often bring to phenomena (Hammersley and Atkinson 1983). Ethnography is not simply description, rather, it is detailed explication. It is about capturing the real movement of experience in the concrete world. Ethnography achieves something which theory and commentary in the majority of cases cannot - namely it presents human experience without minimising it and without making it a passive reflex of structures, organisations and social conditions. 'Human productions are all of a piece, indivisible and always summed. The metal cannot be simply smelted out from the ore of experience in human affairs' (Willis 1978 p. 180).

The use of ethnography is an attempt to ground the understanding of action in empirical evidence. An empirically based social interaction perspective is inductive from particular naturally occurring activities. Ultimately, this will produce descriptions that are accountable to evidence. Situation is crucial to the interpretation of actions but although this is fundamental and to some extent obvious, its importance could easily be overlooked.

Sociologists have long used ethnographic techniques to study work in general. ${ }^{2}$ What is required in $\mathrm{CSCW}$ is an ethnographic analysis of settings that are due to be 'technologized', by which I mean where new forms of information technology are to be implemented. In the case under consideration the settings are where social science teaching and learning takes place. Straightforward ethnographies, such as those developed in the sociology of work, are not sufficient however. What is required is what Button and Dourish (1996) term as a 'technomethodologically' informed approach. This I believe will lead to the successful design and 
implementation of new information technologies in social science teaching settings.

The technomethodological approach departs from the desire to make conspicuous what actors are doing when they organise the activities that they do in particular settings. It draws heavily upon ethnomethodology which turns away from the structures and theorising of traditional sociology, and concentrates instead upon the details of the practices through which action and interaction are accomplished (Button and Dourish 1996).

Ethnomethodology poses the question 'what the devil does the native think they are up to?' (Anderson, Hughes and Sharrock 1989). In the case of CSCW the native is the individual in the setting about to be technologized. Examining settings by technomethodologically informed ethnography is fundamental to the exercise of CSCW if it is to be applied successfully to teaching and learning settings.

The technomethodological approach is underwritten by the work of Garfinkel ${ }^{3}$ who is concerned with that most pervasive sociological question; 'how is it that actions recur and reproduce themselves?' He insists that this orderliness be viewed as arising from within activities themselves and the work being done by the parties to that activity. Garfinkel eschews the traditional sociological strategy of seeking to explain this orderliness and the organisation of social activities by attempting to identify causes and conditions out with the activities themselves (Benson \& Hughes 1983).

Germane to this are Garfinkel's suggestions that it is evident from the availability of empirical specifics that there exists a locally produced order of work's things that make up the enormous domain of organizational phenomena. ${ }^{4} \mathrm{He}$ argues that the classical sociological studies of work, without remedy or alternative, depend upon these phenomena, make use of the domain and ignore it. That the domain is ignored is a systematic feature of the locally produced orderliness of work settings. Therefore the reported phenomena are only inspectably the case, therefore they are unavailable to the art of designing and interpreting definitions, metaphors, models, constructions, types or ideals and cannot be recovered by attempts, no matter how thoughtful to specify an examinable practice by detailing a generality.

The concept of the 'egological organisation' is advanced by Anderson, Hughes and Sharrock (1989) and is an ethnomethodologically informed view of organisations that begins with a bottom-up understanding of them ${ }^{5}$. The conception of the egological organisation departs from an inquiry into the daily or routinized experiences of individuals. The value of this approach is that it places the actor's point of view at the centre of the analysis.

By employing the concept of the egological organisation, they develop the idea of the 'working division of labour'. In Working for Profit they argue that it ought to come as no surprise that actors in work settings see themselves as part of an elaborate working division of labour. From the way that they talk about their work, both to each other and to outsiders it is clear that the notion of a working division of labour is one which they use to interrelate and explicate the things that they see going on about them, on a daily basis and on ordinary occasions. These accounts depict a body of activities marshalled by 'a working division of labour'.

Technomethodology requires a sociological analysis of the organisation of social action and interaction and the organisation of work and work settings. The fullest possible description that captures the essence of the "working division of labour' must be furnished. The thrust of technomethodology is the conception that sociological descriptions of the ways in which people routinely organize their actions and interactions can be furnished and compared to what is or is not possible using technology. In this sense the term 'technomethodology' is an identification of the need for the incorporation of ethnomethodologically informed accounts of the working division of labour that places the actor's perspective at the centre. The object of the ethnographic exercise is therefore to provide what Gertz (1975) terms as 'thick descriptions', which will inform the design and the implementation of the new information technologies. ${ }^{6}$

CSCW can inform the development and implementation of new information technologies that are directed towards teaching and learning environments in the social sciences. I do not wish to argue that the these environments are the same as commercial and industrial settings which so far have been the foci of CSCW. At a generic level university teaching and learning settings are similar insofar as they also require cooperation, coordination and collaboration to accomplish work tasks. And whilst the work carried out in university teaching and learning settings is arguably, often of a more individual nature, $\mathrm{CSCW}$ has attended to the issue of individualistic work. ${ }^{7}$ If we treat the concept of 'doing work' as the active process of 'sense-making' that individuals undertake in settings, then the same kinds of issues that impinge upon actors in commercial work settings are also present in university teaching and learning settings.

\section{Computer Supported Cooperative Work and Teaching in the Social Sciences}

It is certainly the case in Britain that the desire to incorporate technology into higher education teaching and learning has been firmly placed on the higher education agenda. This situation is both desirable and essential to the future development of university education. If in the social sciences we wish to move from using computers as tools, to a scenario where we develop computer based learning 
technologies I believe that it is fundamental to incorporate non-technical knowledge. This will lead to a more circumspect and strategic development of information technology than could be achieved by enthusiasts or technical specialists.

The development of Internet based teaching and learning resources for the social sciences must proceed from clear sets of pedagogical requirements. This is not to argue that in any sense objective sets of criteria that form typologies for effective resources exist. The resource requirements in various settings will be context specific. The role of a CSCW strategy, that is technomethodologically informed, is an attempt to uncover the pedagogical requirements of the Internet computer based resource that is being developed. This is the level of sophistication that is required to develop high quality Internet based teaching and learning resources that will be useful and used in social science departments.

A CSCW approach to the design and implementation of Internet based resources for social science teaching and learning will be liberating. The need for a clear understanding of teaching and learning environments is critical. Technomethodologically informed CSCW, when brought to bear upon the design and implementation of Internet based resources for social science teaching is an attempt to improve what Gurdin (1988) and Bannon \& Harper (1991) term as the 'distinctly random success' of new information technologies.

\section{Bibliography}

Anderson, R., Hughes J. A. \& Sharock, W.W. 1989, Working for Profit: The Social Organisation of Calculation in an Entrepreneurial Firm, Gower Aldershot

Bannon, L. 1989, 'From Human Factors to Human Actores: The Role of Psychology and HCI Studies in Systems Design', Computing Services University College Dublin.

Bannon, L. \& Bodker, S. 1989, 'Beyond the Interface: Encountering Artifacts in Use', Department of Computer Science Aarhus University.

Bannon, L. \& Schmidt, K. 1991, 'CSCW: Four Characters in Search of a Context', Studies in CSCW: Theory, Practice and Design, in J Bowers and S Benford, Elsevier Amsterdam.

Barnard, P. \& Gurdin, J. 1988, 'Command Names', Handbook of Human Computer Interaction, in Helander, M. (ed), North Holland Press Amsterdam.

Beynon, H. 1984, Working for Ford, Penguin London.

Blackler, F. \& Osborne, D. 1987, 'Designs For The Future:
I.T. \& People', British Psychology Society Conference Leicester.

Brooks, R. 1990, 'The Contribution of practitioner case studies to human-computer interaction science', Interaction With Computers, Vol. II No.1 April.

Button, G. \& Dourish, P. 1996 'Technomethodology: Paradoxes and Possibilities', Technical Report EPC-1996101, Rank Xerox, Cambridge.

Carroll, J. 1987, Interfacing Thought: Cognitive Aspects of Human Computer Interaction, MIT Press Cambridge.

Carroll, J. 1989, 'Evaluation, Description and Invention: Paradigms for Human Computer Interaction', Advances in Computers, Vol. 29.

Clark, B.M. \& O’Donnell, S. 1991, 'Computer supported co-operative work', British Telecom Technical Journal, Vol 9 No.1 January pg. 47-56.

Daniel, J. 1993, 'The Challenge of Mass Higher

Education', Studies in Higher Education, Vol 18(2) pp.197203.

Garfinkel, H. 1956, 'Some sociological concepts and methods for psychiatrists', Psychiatric Papers, Vol 6 pp.181-195.

Garfinkel, H. 1967, Studies in Ethnomethodology, Prentice Hall New Jersey.

Garfinkel , H. 1986, Ethnomethodological Studies of Work,Routledge and Kegan Paul London.

Garfinkel, H. 1991, 'Respecification' , in Button, G. Ethnomethodology and the Human Sciences, Cambridge University Press Cambridge.

Gayle, V.J. 1991, 'CSCW and Individual Work: An investigation of work distribution in a London taxi firm', Unpublished Masters Thesis, Department of Sociology University of Lancaster.

Geertz, C. 1975, 'Thick Description' In the Interpretation of Culture, Hutchinson London.

Gray, W. \& Atwood, M. 1988, 'Review of Interfacing Thought', Interfacing Thought: Cognitive Aspects of Human Computer Interaction, in Carroll, J. Interfacing Thought: Cognitive Aspects of Human Computer Interaction, MIT Press Cambridge.

Grief, I. 1988, "Remarks in panel discussion on 'CSCW: What does it mean?' CSCW '88 Proceedings of the conference on CSCW, Sept 26-29 Portland Oregon. 
Hammersley, M. \& Atkinson, P. 1983, Ethnography: Principals in Practice, Tavistock London.

Harper, R. \& Randall, D. 1992, 'Rogues in the Air: An Ethnomethodology of 'Conflict' in Socially Organised Airspace', Technical Report EPC-1992-109, Rank Xerox, Cambridge.

Harper, R., Hughes, J., Randall, D., Shapiro, D., Sharrock, W. (forthcoming), Ordering The Skies, Routledge.

Hirscheim, R, \& Klein, K. 1989, 'Four Paradigms of Information Systems Development', Social Aspects of Computing, Vol 32 No. 10 October pg. 1199-1216.

Hobbes, D. 1988, Doing the Business: Entrepreneurship the Working Class and Detectives in the East End of London, Clarendon Oxford.

Luff, P. \& Heath C. 1990, Collaboration and Control: The Introduction of Multimedia Technology on London Underground", Rank Xerox.

Lyytinen, K. 1989, 'Information Systems Failures: A survey and classification of empirical litterature', Oxford Surveys in Information Technology, Vol.4 pg.257-309.

Pollert, A. 1981, Girls, wives, factory lives, Macmillan, London

Suchman, L. 1987, Plans and Situated Actions: The problem of human machine communication, Cambridge University Press Cambridge.

Thimbleby, H., Anderson, S. \& Whitten, I. 1990, 'Reflexsive CSCW: Supporting long-term personal work', Interacting With Computers, Vol.2 No.3 pg. 330-336.

Willis, P. 1987, Profane Culture, Routledge London.

1 It is important to note that there is no clear or coherent answer to the question 'what is, or was, the goal of HCI?'. One of the more traditional answers to this question is that $\mathrm{HCI}$ intends to provide methods and matrices for evaluating the usability of computer systems. This stems from what can be loosely termed the 'human factors' approach. This is in contrast to cognitive scientists who argue that HCI is a work bench for the application of cognitive psychology to a real problem domain. Computer scientists assert that HCI must help to guide the definition, invention and introduction of new computing tools and environments. This argument is to some extent a product of the exigencies of the computer industry. The point here is to illustrate that $\mathrm{HCI}$ is a diverse discipline with fragmented foci and interests, a feature not often drawn out in HCI literature.

${ }^{2}$ For Example Beynon (1984) examined working at Ford, Hobbs (1987) studied detective work in East London and Pollert (1981) treated the working lives of women in a factory setting.

${ }^{3}$ Garfinkel states that 'the policy is recommended that any social setting be viewed as self-organizing with respect to the intelligible character of its own appearances as either representations of or as evidences-of-a-social-order. Any setting organizes its activities to make its properties as an organized environment of practical activities detectable, countable, recordable, reportable, tell-a-story-aboutable, analyzable - in short, accountable' (Garfinkel 1967 p.33).

${ }^{4}$ See especially Garfinkel (1986 \& 1991).

5 The conception of the egological organisation is motivated by the desire to provide descriptions and analysis, but raises a deep methodological question. Sociological descriptions like other theoretical accounts are thematically constructed. The methodological question at issue in this instance is that employing this approach is an attempt to provide a third person account of first person experience. This does not mean incorporating first person accounts into sociological depictions, rather a sociological re-constitution of that experience is required. The concern is not with particular people's experience, but with the organisation of experience, as it is encountered in social life, as a readily accountable, known and shared schemes of interpretation (Anderson, Hughes and Sharrock 1989).

${ }^{6}$ An example of such an attempt is Harper et al (forthcoming) which is an account of technology and air traffic control as part of an inter-disciplinary attempt to design and implement a technological system.

7 My own work on London Taxi drivers (Gayle 1991) and the work of Thimbleby et al (1990) are two examples.

* Paper presented at the IASSIST/IFDO 1997 Conference, May 6th May 9th Odense, Denmark.

Vernon J. Gayle, Department of Applied Social Science, University of Stirling, Scotland. Email: vg1@ stirling.ac.uk 